\title{
Occurrence of sdh Mutations in German Alternaria solani Isolates and Potential Impact on Boscalid Sensitivity In Vitro, in the Greenhouse, and in the Field
}

\author{
Nicole Metz, ${ }^{\dagger}$ Birgit Adolf, Nicole Chaluppa, Ralph Hückelhoven, and Hans Hausladen \\ Chair of Phytopathology, Technische Universität München, Freising 85354, Germany
}

\begin{abstract}
The fungus Alternaria solani is the main pathogen causing early blight on potatoes (Solanum tuberosum L.). An increase in the development of resistance to the succinate dehydrogenase inhibitor (SDHI) boscalid, one of the main active ingredients for the control of early blight, has been reported. For this study, monitoring data from Germany were collected between 2013 and 2016 and an increase in the occurrence of $A$. solani succinate dehydrogenase (SDH) mutant isolates was observed. In addition to the known point mutations in sdh complex
\end{abstract}

The fungus Alternaria solani (Ellis \& G. Martin) is the main pathogen causing early blight on cultivated potatoes (Solanum tuberosum L.) and is widespread in potato growing areas. Typical symptoms appear on the leaves and include dark brown to black spots with concentric rings and cigar-shaped spores (Rotem 1994). The literature describes yield losses varying from 1 to $60 \%$ for early blight on potatoes in Germany, South Africa, and the United States (Christ and Maczuga 1989; Harrison and Venette 1970; Leiminger and Hausladen 2012; Van der Waals et al. 2003). The pathogen occurs worldwide, has a polycyclic nature, and produces high amounts of secondary inoculum (Campo et al. 2007). Hence, early blight control is considered a challenge for potato farmers. Although farmers follow integrated pest management practices, paying attention to crop rotation, variety selection, and so forth, they often must resort to fungicides to efficiently control the pathogen and protect potato yield. The two main fungicide groups used against early blight in Germany are the strobilurins and the carboxamides. Both the strobilurins as quinone outside inhibitors (QoIs) and the carboxamides as succinate dehydrogenase inhibitors (SDHIs) interfere with the mitochondrial respiration of the fungus. QoIs interact with complex III in the mitochondrial respiration chain, also known as the cytochrome bc1 complex (Bartlett et al. 2002). SDHIs inhibit the activity of succinate dehydrogenase (SDH) in complex II (Kuhn 1984). Because both fungicide groups have a single-site mode of action, there is a higher probability that fungicide resistance will develop compared with fungicides with a multisite mode of action (Kuck and Mehl 2003).

Boscalid was registered for control of early blight on potatoes in the United States in 2003 (Kissling 2003) and in Germany in 2008. Since then it has been applied on a regular basis. In 2009, the first loss of $A$. solani sensitivity to boscalid was detected in Idaho (Wharton et al. 2012). In addition to the partial loss of $A$. solani fungicide sensitivity to QoIs (Leiminger et al. 2014; Odilbekov et al. 2016; Pasche et al. 2004), reports of resistance to the SDHI boscalid have also increased (Gudmestad et al. 2013; Landschoot et al. 2017a). The reasons for this increasing resistance to SDHIs are specific point mutations in the SDH complex (complex II). In this complex, SDHIs

${ }^{\dagger}$ Corresponding author: N. Metz; nicole.metz@tum.de

The author(s) declare no conflict of interest.

Accepted for publication 28 May 2019.

@) 2019 The American Phytopathological Society are able to bind to three subunits ( $\mathrm{SdhB}, \mathrm{SdhC}$, and $\mathrm{SdhD}$ ) (Horsefield et al. 2006). Five point mutations in complex II have been described for A. solani (Mallik et al. 2014): two in each of the SdhB and SdhD genes and one in the SdhC gene. In subunit B, mutations H278R and H278Y are known thus far. The background of these mutations is an amino acid exchange from histidine to arginine or tyrosine, respectively. For subunit C, a substitution of histidine to arginine at amino acid position 134 leads to the H134R mutation, and the same substitution at position 133 in the SdhD gene leads to the H133R mutation. A second point mutation in subunit $\mathrm{D}$ at position 123 with an exchange of aspartate to glutamic acid leads to the D123E mutation. Until 2013, the detected mutants carried only one mutation, but double mutations were also found in the United States and Belgium in 2014 and 2016, respectively (Landschoot et al. 2017a; Mallik et al. 2014).

Thus far, the increasing resistance of $A$. solani to the SDHI boscalid has only been shown in vitro and in greenhouse trials, and there have been no reports on experiments with mutated isolates and the fungicide applied in the field under real growing conditions. In this study, all three stages of SDHI fungicide sensitivity testing were performed, including in vitro, in the greenhouse, and in field trials. Furthermore, field isolates of $A$. solani were collected from across Germany between 2013 and 2016. These isolates were screened for different SDH mutations and used for monitoring. The goal of this study was to obtain a broader picture of the dynamic occurrence of SDH mutants in Germany and to produce direct evidence for the loss of boscalid sensitivity of naturally occurring SDH mutant isolates in the field.

\section{Materials and Methods}

Isolate sampling and detection of mutations for monitoring. Infected potato leaves with typical early blight symptoms were collected from several locations across Germany (Fig. 1) between 2013 and 2016 (22, 28, 34, and 26 locations, respectively). Up to 15 isolates were obtained from each location to assess the predominant distribution of SDH wild-type and SDH mutant isolates. The locations varied from year to year, depending on the participation of farmers and public agencies in the monitoring program, and no field was sampled twice, as a result of crop rotation in these regions.

To obtain single spore isolates from the infected leaves, small portions of plant tissue from the edge of the typical symptoms were transferred to SN agar (SNA: $0.2 \mathrm{~g} /$ liter of glucose, $0.2 \mathrm{~g} /$ liter of sucrose, $0.5 \mathrm{~g} /$ liter of $\mathrm{MgSO}_{4}-7 \mathrm{H}_{2} \mathrm{O}, 0.5 \mathrm{~g} /$ liter of $\mathrm{KCl}, 1 \mathrm{~g} /$ liter of $\mathrm{KH}_{2} \mathrm{PO}_{4}, 1 \mathrm{~g} /$ liter of $\mathrm{KNO}_{3}, 22 \mathrm{~g} /$ liter of agar, and $600 \mu \mathrm{l} /$ liter of $1 \mathrm{M} \mathrm{NaOH}$ ) directly after surface sterilization with $3 \% \mathrm{NaOCl}$ for $3 \mathrm{~min}$. After 4 to 5 days of incubation at $21^{\circ} \mathrm{C}$ and under nearultraviolet (UV) light (12 h/12 h), a single spore of A. solani was 
picked and transferred to a new SNA plate. The fungus was initially identified on the basis of morphological characteristics and spore size (Rotem 1994). In addition, some isolates were randomly identified as A. solani via sequencing of the calmodulin gene and the RNA polymerase second-largest subunit according to Landschoot et al. (2017b). The single spore isolates were cultivated for 7 to 10 days on SNA and then transferred to $\mathrm{V} 8$ agar $\left(2 \mathrm{~g} / \mathrm{liter}\right.$ of $\mathrm{CaCO}_{3}, 15 \mathrm{~g} / \mathrm{liter}$ of agar, $200 \mathrm{ml}$ of legume juice, and $800 \mathrm{ml}$ of distilled $\mathrm{H}_{2} \mathrm{O}$ ). V8 agar induces sufficient mycelium growth to obtain enough DNA to screen for quinone outside (Qo) and SDH mutations.

Genomic DNA was extracted from mycelia cultivated on V8 agar for 14 days under near-UV light at $21^{\circ} \mathrm{C}$ using a cetyl trimethylammonium bromide protocol. DNA content was measured with a NanoDrop spectrophotometer and then adjusted to $20 \mathrm{ng} / \mu \mathrm{l}$ for the Qo and $\mathrm{SdhC} / \mathrm{D}$ screening and to $40 \mathrm{ng} / \mu \mathrm{l}$ for $\mathrm{SdhB}$. Finally, Sdh complex II and Qo complex III of the isolates were sequenced with different primers (Table 1) by LGC Genomics GmbH to detect mutations in these subunits.

In vitro assessment of SDHI sensitivity. An in vitro plate assay was performed to determine the sensitivity of $A$. solani isolates to the SDHI boscalid, based on germination of conidia on fungicideamended agar (Olaya et al. 1998). Because of problems with dissolving the pure boscalid at higher concentrations, the commercial

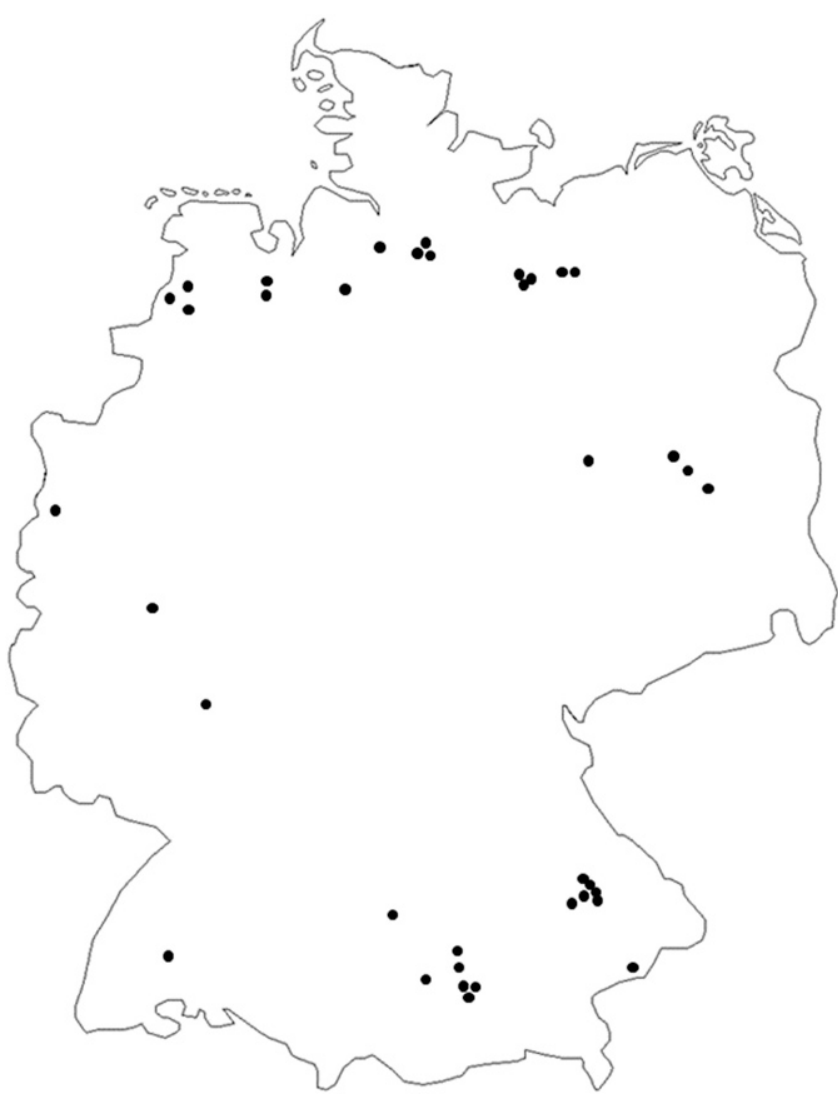

Fig. 1. Geographical distribution of all sampled locations in Germany between 2013 and 2016. Up to 15 isolates were obtained for each location and year. product Cantus (BASF; $500 \mathrm{~g}$ of boscalid $/ \mathrm{kg}$ ) was used for the tests For the highest concentration of the active ingredient, Cantus was dissolved in distilled $\mathrm{H}_{2} \mathrm{O}$ to a final concentration of $100 \mu \mathrm{g}$ of boscalid $/ \mathrm{ml}$ in the agar. For the lower concentrations, a stock solution was prepared and added to the medium in different amounts. A new stock solution was used for each experiment in order to prevent degradation of the compounds. The diluted compounds were added to $1.5 \%$ water agar after autoclaving $\left(121^{\circ} \mathrm{C}, 20 \mathrm{~min}\right)$. Sensitivity was determined by comparing spore germination on $1.5 \%$ water agar with a concentration of $0,0.1,1,10$, and $100 \mu \mathrm{g} / \mathrm{ml}$ of the active ingredient (boscalid).

To obtain a spore suspension of each $A$. solani isolate, sterile distilled water was added to the fully overgrown SNA plates and conidia were scratched off with a sterile object slide. The conidial suspension was adjusted to $1 \times 10^{4}$ conidia/ml using a hemocytometer. Then, $130 \mu \mathrm{l}$ of the suspension of each isolate was added to two replicate plates of each fungicide dilution using a sterile Drigalski spatula. After the plates were incubated in the dark for $5 \mathrm{~h}$ at $26^{\circ} \mathrm{C}$, germination of 100 conidia was assessed by using a binocular microscope at $\times 4.5$ magnification (Zeiss). A conidium was considered germinated if the germ tube was at least half the length of the conidium. The $\mathrm{EC}_{50}$ value, which shows the fungicide concentration that effectively reduces germination by $50 \%$ relative to the untreated control, was determined for each isolate according to Pasche et al. (2004). In total, 47 isolates were evaluated. More isolates were initially included but had to be excluded because of limited growth and spore germination. The calculated $\mathrm{EC}_{50}$ values were divided into three groups according to Gudmestad et al. (2013): sensitive $(<5 \mu \mathrm{g} / \mathrm{ml})$, moderately resistant (5 to $20 \mu \mathrm{g} / \mathrm{ml}$ ), and highly resistant ( $>20 \mu \mathrm{g} / \mathrm{ml})$.

In vivo assessment of SDHI sensitivity in the greenhouse. The efficacy of boscalid was assessed against a subset of three SDH wildtype and $13 \mathrm{SDH}-$ mutated $A$. solani isolates in greenhouse trials. More isolates were tested but were excluded from further studies as a result of very low infection rates during the trials. The selected A. solani mutant isolates had mutations in different parts of the SDH complex: two SDHB-H278R, four SDHB-H278Y, five SDHCH134R, and two SDHC-H134Q mutants were tested. Isolates were cultivated as described for the in vitro test. For this experiment, the potato cultivar Kuras was used, which is known to be susceptible to early blight. Boscalid concentrations of $0.1,1,10$, and $100 \mu \mathrm{g} / \mathrm{ml}$ were used. An untreated but $A$. solani-infected control and an untreated noninfected control were included in the trial. The 6-weekold plants were cut to three uniformly sized leaflets and all of the other leaves were removed to provide a standard starting point for all assessments. One day before inoculation with the pathogen, the plants were sprayed preventively with the different fungicide concentrations. Three biological replicate plants per variation were used and the whole experiment was repeated twice with all five concentrations and once only with the highest dose of boscalid $(100 \mu \mathrm{g} / \mathrm{ml})$. For the final analysis, the experimental data from the $100 \mu \mathrm{g} / \mathrm{ml}$ boscalid treatment were used, because this concentration showed the greatest differentiation. Each fungicide was applied until runoff in order to evenly wet the whole plant. Twenty-four hours after fungicide application, spore suspensions of the A. solani isolates were sprayed on the upper side of the leaves with a chromatography sprayer, also until runoff. The spore density was between 6.8 and $10 \times 10^{4}$ spores $/ \mathrm{ml}$. After inoculation, the plants were transferred to greenhouse chambers with a temperature of $20^{\circ} \mathrm{C}$ and an air humidifier to generate nearly $100 \%$ relative humidity for $48 \mathrm{~h}$. A high-pressure mist blower

Table 1. Primers used to detect succinate dehydrogenase (SDH) and quinone outside inhibitor (QoI) mutations in Alternaria solani isolates

\begin{tabular}{lllll}
\hline Target & \multicolumn{1}{c}{ Species } & \multicolumn{1}{c}{ Primer name $^{\mathbf{z}}$} & \multicolumn{1}{c}{ Primer sequence } & \multicolumn{1}{c}{ Reference } \\
\hline SdhB & A. solani & SdhB-F, SdhB-R & ATGGCCTCCATACGCGCTTT & Mallik et al. (2014) \\
& & & CTAGGTGAAGGCCATGCTCTT & \\
SdhC & A. solani & SdhC-F1, SdhC-R2 & ATGGCTTCTCAGCGGGTATTTCAGC TCCATCCAGTGCGGATAACC & Mallik et al. (2014) \\
SdhD & A. solani & SdhD-F1, SdhD-R2 & ATGGCCTCCGTCATGCGT CCTCGGTGATACCAACATCGTTTGTC & Mallik et al. (2014) \\
QoI & A. solani (genotype I) & As-Gf, As-Gr & CGGGGACTAATATTTTGATA TGTTATTTAACCAAGAATGAAA & Leiminger et al. (2014) \\
\hline
\end{tabular}

${ }^{\mathrm{z}} \mathrm{F}=$ forward and $\mathrm{R}=$ reverse. 
was run constantly for the first 2 days and subsequently only started automatically if the air humidity was lower than $70 \%$. To determine the disease severity, the percentage of infected leaf area was visually assessed according to the scheme of Granovsky and Peterson (1954) for each of the three leaflets. Disease severity rating was carried out 5,7 , and 10 days after inoculation.

Assessment of SDHI sensitivity in the field. A field trial was carried out with a targeted inoculation with specific $A$. solani isolates for the first time in 2016 and 2017 in Freising, Germany. The aim was to obtain more detailed information about the predominance of SDH wild types or SDH mutants and their influence on the efficacy of SDHI fungicides. Fields were inoculated with either an SDHmutated or nonmutated isolate to get a direct comparison between a field with a mostly SDH-mutated or wild-type A. solani population. The trial was treated with Cantus (0.2 liters/ha) at 14-day intervals with a backpack sprayer.

Trial setup for 2016 and 2017. A randomized design with four replicates per variation was used for the trial setup. The plot size was $18 \mathrm{~m}^{2}$ with six rows $(0.75 \mathrm{~m}$ between each row). The trial was carried out with the potato cultivar Lady Amarilla from the early maturity group, which is known to be very susceptible to early blight. Planting dates were 10 May 2016 and 11 May 2017, and flowering time was at the end of June for both years. There was no artificial inoculation with $A$. solani on the chosen fields prior to the experiment. All plots were fertilized and cultivated according to general agricultural practice. To achieve a targeted infection with a specific SDHmutated isolate, an artificial kernel inoculation was carried out as described below. A noninfected and nonfungicide-treated variation was also included as a reference to the inoculated controls.

Inoculation with specific $\boldsymbol{A}$. solani isolates. In this trial, three different $A$. solani isolates were used for kernel infection (Table 2): two with the mutation H134R in subunit $\mathrm{C}$ of the SDH complex and one SDH wild-type isolate. All three isolates also have the F129L mutation in complex II (QoI mutation).

SNA plates were used to cultivate all three different isolates under near-UV light $(12 \mathrm{~h} / 12 \mathrm{~h})$ at $21^{\circ} \mathrm{C}$ for 2 weeks. To generate the kernel inoculum, an autoclavable bag was filled with $150 \mathrm{~g}$ of barley kernels and $60 \mathrm{ml}$ of distilled water and closed with a buckler and rubber band. The bags were autoclaved, allowed to cool down, shaken to mix the kernels, and reautoclaved. Then, half of a fully covered 2week-old SNA plate was used to infect the kernels with the fungus. The SNA plate was cut into small pieces $(0.5$ to $1 \mathrm{~cm})$ to simplify an even distribution of the fungus in the bag. The bags were incubated for 4 weeks under near-UV light $(12 \mathrm{~h} / 12 \mathrm{~h})$ at $21^{\circ} \mathrm{C}$ to provide optimum conditions for fungal growth. To guarantee a consistent illumination, the bags were kneaded again and turned around after 2 weeks. Approximately 6 weeks after planting, the inoculated kernels were spread equally between the potato rows $\left(5 \mathrm{~g} / \mathrm{m}^{2}\right)$. Inoculation was carried out on 29 June 2016 and 21 June 2017.

The field was divided into four parts and each part was inoculated with either the mutated isolates 687 _ 1 or 628 , the wild-type isolate 615 , or a noninoculated control. To minimize intermixing of the isolates by wind or rain splash, an additional 4-m plot was included between each inoculation. Within each isolate, a randomized design with four replicates was used.

Fungicide treatments. Fungicide treatment with Cantus $(0.2$ liters/ha) started in the middle of July and was then applied at 2-week intervals. There was also an untreated control for each of the three isolates used.

All plots were sprayed with herbicides and insecticides according to good agricultural practice. The whole field was also independently treated weekly with oomycete active fungicides (alternating 0.6 liters of Revus/ha [250 g of mandipropamid/liter] and 0.2 liters of Ranman/ha [400 g of cyazofamid/liter]) against late blight (Phytophthora infestans) to ensure that early blight was the predominant disease on the potatoes.

Disease assessment. With the occurrence of the first symptoms (15 July 2016 and 17 July 2017), disease progression was assessed at 7- to 10-day intervals until the plants died. The last assessment for further calculations was performed on 31 August for both years. Each assessment was performed by the same person. For the assessment, 10 plants per plot were rated for disease progression of A. solani. To exclude the influence of surrounding plots, only the two rows in the middle of each plot were used. A scheme published by Granovsky and Peterson (1954) was used to determine the percentage of infected leaf area. The rating was done for all four replicates. The relative area under the disease progress curve (rAUDPC) was evaluated at the end of the experiment. Therefore, the area under the disease progress curve (AUDPC) was first calculated according to Shaner and Finney (1977). The rAUDPC was then the calculated AUDPC for the specific variant relative to the AUDPC with a disease severity of $100 \%$ at each time point.

Isolate sampling from the field trial. Leaf samples were taken during the season to ensure that the assessed A. solani infection on the leaves was traced back to the kernel inoculum with its specific SDH region. Potato leaves with symptoms were placed on SNA for 4 to 5 days (growing conditions as described above). After sporulation, single spore isolates of $A$. solani were isolated, cultured on SNA and V8 agar, and finally screened for mutations in subunits $\mathrm{B}, \mathrm{C}$, and $\mathrm{D}$ of the SDH complex to prove the presence of the inoculated mutant or wild type.

Statistical analysis. All data sets were checked for variance homogeneity (Levene test). If the variances were equal, an analysis of variance with the less conservative Tukey $b$ test $(\alpha=0.05)$, instead of the common Tukey honest significant difference test, was then performed. If no variance equality could be determined, a Dunnett T3 test $(\alpha=0.05)$ was used. All statistical analysis was performed using SPSS Statistics 23 software.

\section{Results}

Occurrence of SDH mutants in Germany and a new mutation. During monitoring of genomic polymorphisms in $S D H$ genes of A. solani isolates, the first SDH mutations were detected in 2013 . SDHB-H278Y and SDHC-H134R mutant isolates were found in 5 and $9 \%$ of the observed locations, respectively. In 2014, no SDHB-H278Y mutants were observed, and SDHC-H134R was detected in $7 \%$ of locations. In addition, a previously unknown mutation was recorded in SDH subunit $\mathrm{C}$. The amino acid histidine at position 134 was replaced with glutamine (SDHC-H134Q). In 2015, the number of locations with SDHB-H278Y and SDHCH134R mutations greatly increased (18 and 62\%, respectively) and SDHB-H278R and SDHD-D123E mutants were found in one location each. In 2016, no SDHC-H134Q or SDHD-D123E mutants were detected. The genotype SDHC-H134R was predominant in 2016 (69\%), followed by SDHB-H278Y (35\%) (Table 3).

Sensitivity of selected isolates to boscalid in vitro. $\mathrm{EC}_{50}$ values were determined in order to assess sensitivity to the commercial fungicide Cantus in vitro. A total of $20 \mathrm{SDH}$ wild-type, $11 \mathrm{~B}-\mathrm{H} 278 \mathrm{Y}, 14$ C-H134R, and two C-H134Q isolates were tested.

All 20 wild-type isolates were sensitive $(<5 \mu \mathrm{g} / \mathrm{ml})$ to Cantus. The average $\mathrm{EC}_{50}$ value for the tested wild-type isolates was 0.19 (data not shown). In contrast, all SDHB-H278Y isolates showed moderate ( 5 to $20 \mu \mathrm{g} / \mathrm{ml}$ ) or high ( $>20 \mu \mathrm{g} / \mathrm{ml}$ ) resistance to the fungicide. Similarly, only one of 14 SDHC-H134R isolates was categorized as sensitive, whereas the others were moderately or highly resistant to Cantus. One SDHC-H134Q isolate was sensitive and the other was moderately resistant to Cantus in vitro (Fig. 2).

Sensitivity of selected isolates to boscalid in the greenhouse. Subsequent to the sensitivity testing in vitro, greenhouse trials were performed using the pure active ingredient of Cantus, boscalid. Three SDH wild-type isolates and two SDHB-H278R, four SDHB-H278Y, five SDHC-H134R, and two SDHC-H134Q mutant $A$. solani isolates

Table 2. Isolates used for the field trial

\begin{tabular}{lccc}
\hline Isolate & Qo mutation $^{\mathbf{y}}$ & SDH mutation & Subunit \\
\hline 615 & F129L & $-^{\mathrm{z}}$ & - \\
628 & F129L & H134R & Subunit C \\
$687 \_1$ & F129L & H134R & Subunit C \\
\hline
\end{tabular}

${ }^{\mathrm{y}} \mathrm{Qo}=$ quinone outside and $\mathrm{SDH}=$ succinate dehydrogenase.

${ }^{\mathrm{z}}$ Dashes indicate no mutation. 
were tested in at least two independent experimental setups with three plants per treatment. After inoculation with wild-type isolates, boscalid proved $99 \%$ efficient on average. Boscalid was somewhat less efficient in reducing leaf symptoms after inoculation with the SDHB-H278R and SDHC-H134Q mutants (69 and 88\% efficacy, respectively), but the effect was not statistically significant compared with the efficacy of wild-type inoculations (99\%). In contrast, boscalid did not efficiently protect the leaves after inoculation with either the SDHB-H278Y mutant (40\% efficacy) or the SDHCH134R mutant (51\% efficacy) compared with the wild-type inoculations (99\% efficacy) (Fig. 3).

Sensitivity of some isolates to SDHI fungicide Cantus in the field. Potato inoculation was performed in the field in 2016 and 2017. Different plots of the trial were inoculated with wildtype A. solani isolates or SDHC-H134R mutants (two different isolates). Isolates with the SDHC-H134R mutation were used, because this was the most frequent mutation observed in the monitoring. Comparison of the rAUDPC values of the different untreated controls in 2016 (Fig. 4) showed that there were significant differences between the natural infection (0.31), the wild type (0.38), and the SDHC-H134R2 inoculation (0.48). The untreated control of the SDHC-H134R1 inoculation (0.33) was not significantly different from the natural infection.

A comparison of the effects of the fungicide treatments for each artificial inoculation in 2016 showed a significant reduction in rAUDPC values after inoculation with the wild type (0.22) and SDHC-H134R1 (0.21) compared with the respective untreated controls (0.38 and 0.33 , respectively). In contrast, no differences in rAUDPC values were recorded between the untreated control (0.48) and the Cantus treatment after inoculation with SDHCH134R2 (0.47), and rAUDPC values were also significantly higher compared with the other two genotypes.

In 2017, all inoculated control variants showed significantly higher rAUDPC values than the naturally infected control $(0.29)$
(Fig. 5). Furthermore, a significantly lower rAUDPC was detected for the wild-type untreated control compared with the two mutant untreated controls, which behaved similarly to each other.

The treatment with Cantus was effective in reducing rAUDPC values in the wild-type inoculation in 2017 (from 0.38 to 0.21 ). In contrast, Cantus did not cause a significant reduction in rAUDPC values after inoculation with the selected SDHC mutants, SDHC-H134R1 (from 0.48 to 0.40 ) and SDHC-H134R2 (from 0.49 to 0.48 ).

\section{Discussion}

After introduction of specific fungicides into the market, fungicide resistance often occurs quickly and regionally in plant pathogenic fungi (Pasche et al. 2004). The subsequent discovery that target site mutations in pathogenic fungi are associated with loss of fungicide sensitivity in vitro and in greenhouse experiments (Landschoot et al. 2017a; Leiminger et al. 2014; Rehfus et al. 2018) seems to explain fungicide resistance in practice. However, only a limited amount of direct evidence exists showing that mutant strains collected from the field can indeed cause plant disease in the field, which cannot be controlled by the respective fungicide.

To obtain an overview of the presence and accumulation of A. solani SDH mutants with possible fungicide resistance in Germany, isolates were collected over the years 2013 to 2016 and amplicons of their SDH genes were resequenced. Previously described SDH mutations (Mallik et al. 2014) were indeed detected, and a new mutation in SDH subunit C (SDHC-H134Q) was also identified. This particular amino acid exchange has not been reported for other plant pathogenic fungi, according to the Fungicide Resistance Action Committee (2015). The SDHC-H134Q genotypes were less frequent than the SDHC-H134R genotypes and did not appear to accumulate over time, but they seemed to disappear again from the populations monitored in 2016. This is possibly explained by the fact that the SDHC-H134Q mutants tested did not show a substantial loss in fungicide sensitivity. Hence, other more aggressive mutants might have

Table 3. Number of locations with the respective genotype from 2013 to 2016

\begin{tabular}{cccccccccc}
\hline Year & Locations $(\boldsymbol{n})$ & Isolates $(\boldsymbol{n})$ & WT $^{\mathbf{z}}$ & SDHB-H278R & SDHB-H278Y & SDHC-H134R & SDHC-H134Q & SDHD-D123E & SDHD-H133R \\
\hline 2013 & 22 & 137 & 100 & 0 & 5 & 9 & 7 & 0 & 0 \\
2014 & 28 & 67 & 93 & 0 & 0 & 6 & 0 \\
2015 & 34 & 196 & 44 & 3 & 18 & 62 & 3 & 3 \\
2016 & 26 & 325 & 35 & 4 & 35 & 69 & 0 & 0 & 0 \\
\hline
\end{tabular}

${ }^{\mathrm{z}}$ Wild type (WT) and succinate dehydrogenase (SDH) values are given in percentages.

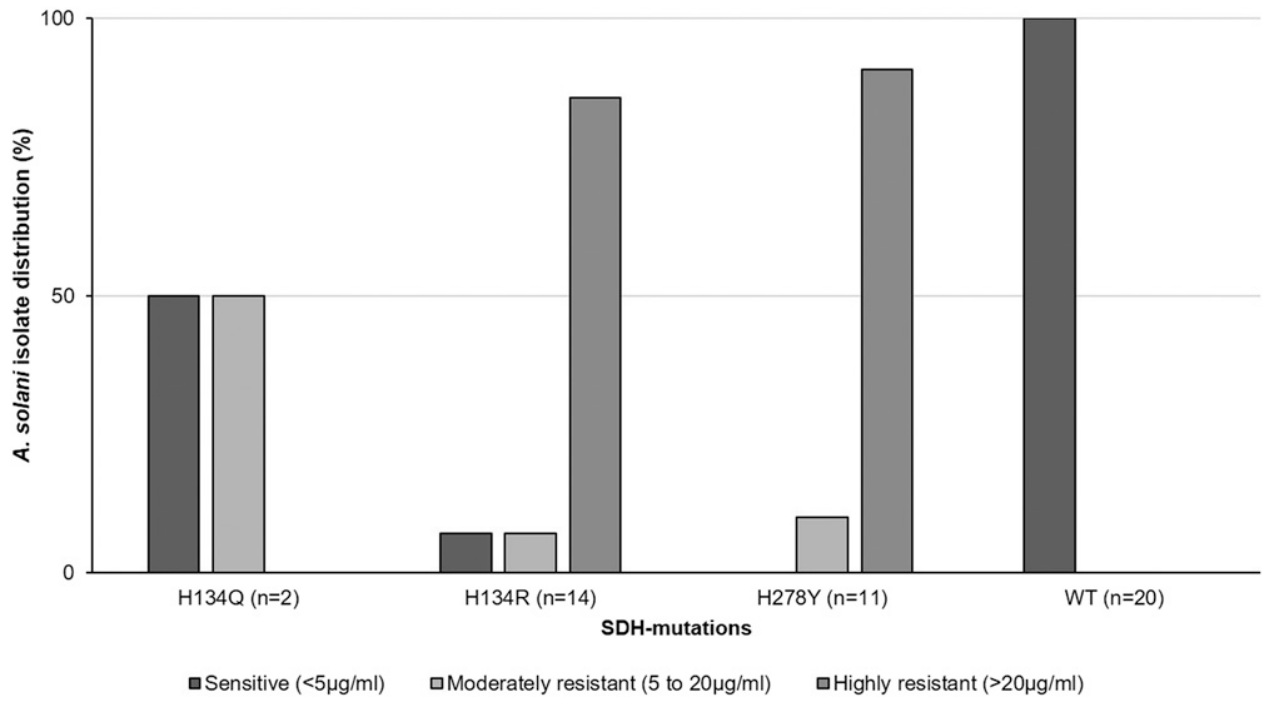

Fig. 2. Distribution of 47 Alternaria solani isolates with either no (WT = wild type) or one mutation (B-H278Y, C-H134Q, and C-H134R) in succinate dehydrogenase (SDH) according to their sensitivity to the active ingredient boscalid in vitro. According to their determined $\mathrm{EC}_{50}$ values, the isolates were placed in the sensitive $(<5 \mu \mathrm{g} / \mathrm{ml})$, moderately resistant $(5$ to $20 \mu \mathrm{g} / \mathrm{ml})$, or highly resistant $(>20 \mu \mathrm{g} / \mathrm{ml})$ group. $\mathrm{n}=$ number of tested isolates. 
outcompeted these mutants over time. Accordingly, the frequency of less fungicide-sensitive SDHC-H134R and SDHB-H278Y mutants appeared to constantly increase over time. More generally, the monitoring of $A$. solani isolates in Germany clearly shows an increasing amount of SDH mutants between 2014 and 2016. The SDHCH134R mutation was found most frequently each year in Germany. In the United States, the most common mutation in the A. solani $S D H$ gene is year dependent. The SDHB-H278Y mutation was predominant in 2011 and 2012, whereas the mutation found most frequently in 2015 was SDHC-H134R (Bauske et al. 2018a), as in the results here. For disease management and disease control practice, it is important to know what effects the spreading of these individual SDH mutations have on fungicide efficacy. Rehfus et al. (2018) showed for Zymoseptoria tritici that some SDH mutations (B-N225T,
B-T268I, C-T79N, C-W80S, C-N86S, C-H152R, C-V166M) affect SDHI fungicide efficacies depending on their different active ingredients. To generate a more complete picture of the fungicide efficacy of one of the most used fungicides to control early blight in Germany, the efficacy of Cantus and its active ingredient boscalid was tested in different systems. The presence of SDH mutations led to reduced fungicide efficacy in all systems tested.

Wharton et al. (2012) showed frequent insensitivity of $A$. solani isolates of an unknown genotype against boscalid. For the German A. solani isolates, different levels of sensitivity were measured. Concerning the $\mathrm{EC}_{50}$ values in vitro, the majority of the SDHC-H134R mutants were classified as highly resistant to boscalid and only one of 15 isolates retained sensitivity. For the SDHC-H278Y mutation, only one of nine isolates was moderately resistant and the rest proved

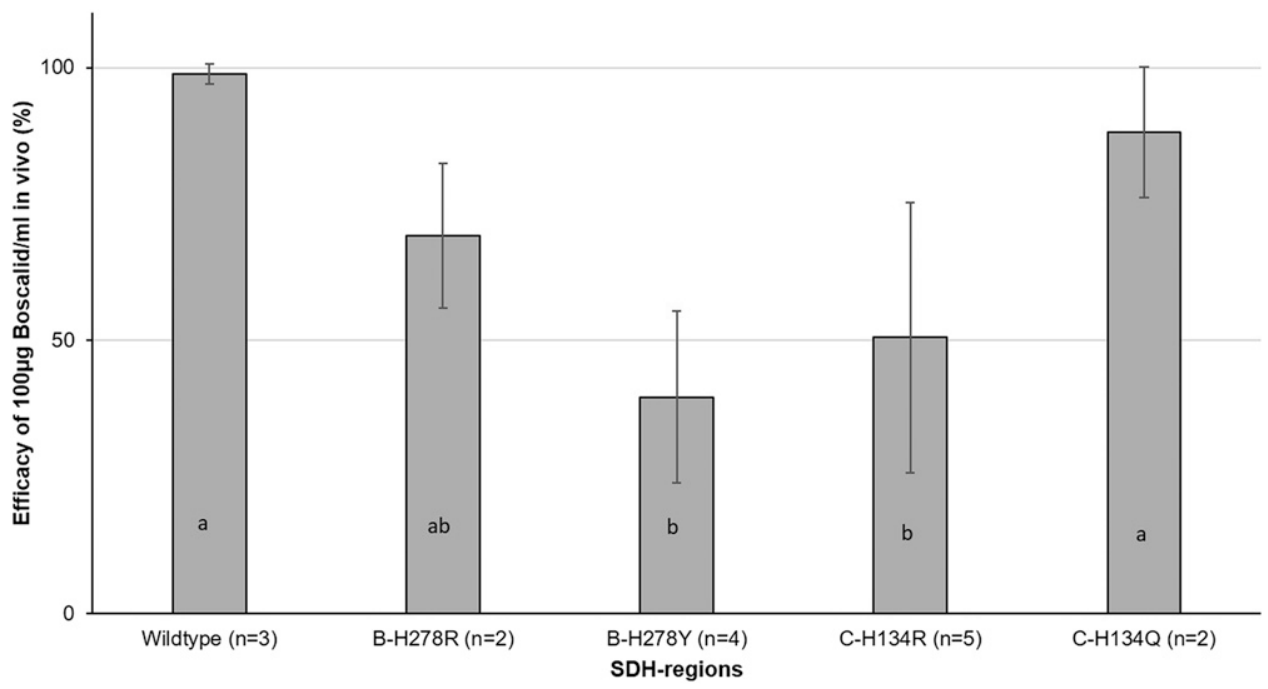

Fig. 3. Fungicide efficacy of $100 \mu \mathrm{g}$ of boscalid/ml in greenhouse trials. Efficacy is given in percentages. The succinate dehydrogenase (SDH) wild type and SDHB-H278R, SDHBH278Y, SDHC-H134R, and SDHC-H134Q isolates were tested. Each isolate was tested in at least two independent experimental setups with three plants for each treatment, using the potato cultivar Kuras. Bars marked with the same letter are not significantly different according to the post hoc Dunnett T3 test $(\alpha=0.05)$. Error bars show the standard deviation. $n=$ number of tested isolates.

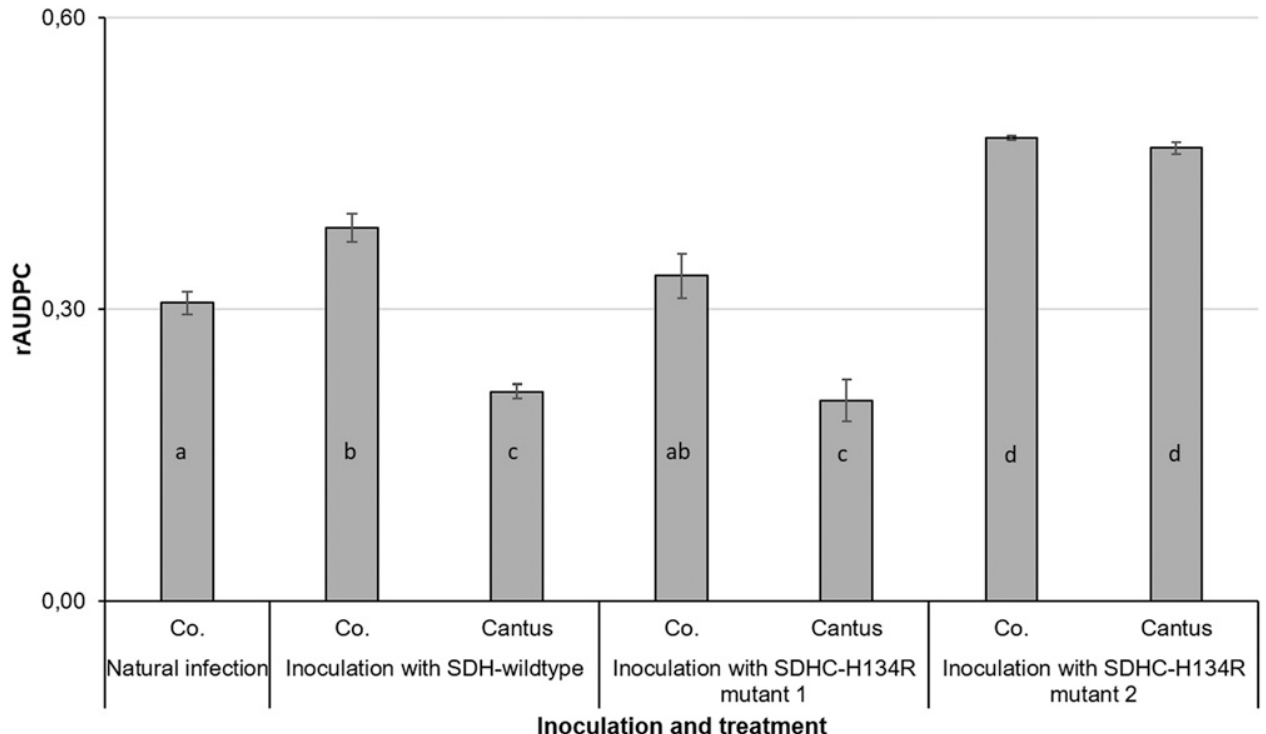

Fig. 4. Relative area under the disease progress curve (rAUDPC) values of three separate field inoculations with different Alternaria solani isolates-one succinate dehydrogenase (SDH) wild type (isolate 615) and two SDHC-H134R mutants (isolate 628 is SDHC-H134R mutant 1 and isolate 687_1 is SDHC-H134R mutant 2)-and a natural infection in the field trial in 2016, using potato cultivar Lady Amarilla. For each inoculation, the mean rAUDPC values (calculated from four repetitions) of the untreated control (Co.) and the treatment with the SDHl fungicide Cantus $(500 \mathrm{~g}$ of boscalid $/ \mathrm{kg}$ ) are shown. Bars marked with the same letter are not significantly different according to the post hoc Dunnett T3 test $(\alpha=0.05)$. Error bars show the standard deviation. 
highly resistant. Thus, for the cases tested in vitro (except for SDHCH134Q mutants, as already discussed), the presence of SDH mutations in German A. solani isolates is associated with very low sensitivity to boscalid. In contrast, only a few SDH mutant isolates were classified as moderately resistant. These results are comparable to those reported by Gudmestad et al. (2013). These authors also showed that highly boscalid-resistant isolates occurred more frequently than moderately resistant isolates. However, the SDH genotypes were unknown at this time. Landschoot et al. (2017a) reported similar results for Belgian $A$. solani isolates with individual genotypes (SDHB-H278R/Y, SDHC$\mathrm{H} 134 \mathrm{R})$ in vitro. As in the results here, the different mutations led to highly resistant isolates in most cases. Several other plant pathogenic fungi, such as Corynespora cassiicola and Botrytis cinerea (Teramoto et al. 2017; Yin et al. 2011), also exhibit different levels of sensitivity to boscalid.

Next, the efficacy of $100 \mu \mathrm{g} / \mathrm{ml}$ of boscalid was tested in greenhouse whole-plant inoculation trials in order to include the interaction with the potato plant and to get closer to field conditions. Greenhouse trials have the advantage of being conducted with relatively low effort and a higher possible throughput in the number of isolates compared with field trials. Gudmestad et al. (2013) showed that when potato plants were treated with $100 \mu \mathrm{g} / \mathrm{ml}$ of boscalid, the fungicide showed significantly reduced efficacy after prior inoculation with $A$. solani isolates, which were of an unknown genotype but were moderately or highly resistant to boscalid in vitro. In these trials, the boscalid treatment showed nearly $100 \%$ efficacy for the $\mathrm{SDH}$ wild-type inoculations. For the SDH mutant genotypes, little efficacy was found against the frequently occurring SDHB-H278Y and SDHC-H134R genotypes but there was still good efficacy against the less frequent SDHB-H278R and SDHC-H134Q mutants. Thus, these in vivo results support the assumption that there is no strong selective advantage for the pathogen to have these mutations (SDHB-H278R and SDHC-H134Q), explaining their low frequencies and transient appearance. Vice versa, the strong loss of boscalid sensitivity in the SDHB-H278Y and SDHC-H134R genotypes may explain their increasing frequency in the German population.

A study from Rehfus et al. (2018) indicates the influence of different SDH mutations in Z. tritici on SDHI fungicide efficacy in greenhouse trials. In this study, different SDH mutations (B-N225T, B-T268I, C-T79N, C-W80S, C-N86S, C-H152R, C-V166M) led to different levels of fungicide sensitivity. Rehfus et al. (2018) also showed the influence of different SDH mutations on the efficacy of different SDHI fungicides. Future work based on isolates reported here might show whether $A$. solani, too, shows SDHI-specific and genotype-specific loss of fungicide sensitivity.

Field trials are considered the best way to observe the interactions between the pathogen, the plant, the environment, and a fungicide in an isolate-specific way. To ensure specific $A$. solani infection with either the SDH wild type or SDHC-H134R mutants in the field, the trials were inoculated with infected barley kernels. In this way, successful infections with $A$. solani can be achieved even if the weather conditions are not favorable for the fungus. Kernel inoculation has the advantage of being a natural means of infection. Typically, A. solani overwinters in the soil and initially infects the lowest leaves first. It then develops from one leaf level to the next by wind and rain splash. With the infected barley kernels lying on the ground between the rows, the infection with $A$. solani also started on the lower leaves and developed like a natural infection during the season. In general, inoculation leads to higher pathogen pressure in the field, which can help to give a clearer picture of fungicide performance. However, this also limits comparison between field trials and fields in practice. Nevertheless, field trials may improve prediction of the effect of the increasing frequency of SDH mutants in the fields on future fungicide efficacy.

In both 2016 and 2017, the fungicide efficacy of boscalid in the field trials was significantly reduced in the mutant inoculations compared with the wild-type inoculation. Furthermore, the efficacy of boscalid in 2016 was significantly reduced in one of the mutant inoculations compared with the wild-type inoculation. After inoculation with SDHC-H134R-1, no reduced efficacy of Cantus was observed in 2016, although the same isolate had shown boscalid resistance in vitro and in the greenhouse. However, in untreated controls, the corresponding rAUDPC did not reach levels higher than those with natural infection. It is therefore concluded that inoculation with SDHC-H134R-1 failed to increase the infection pressure and that the fungicide might have acted on the natural background infection in this case. For 2017, a significant reduction in the efficacy of Cantus was observed for both mutant inoculations compared with the wildtype inoculation. These results show that Cantus was not efficient in the control of early blight under high infection pressure from SDHmutated A. solani isolates. When inoculation with SDHC-H134R succeeded in the field, a near total loss of fungicide efficacy was

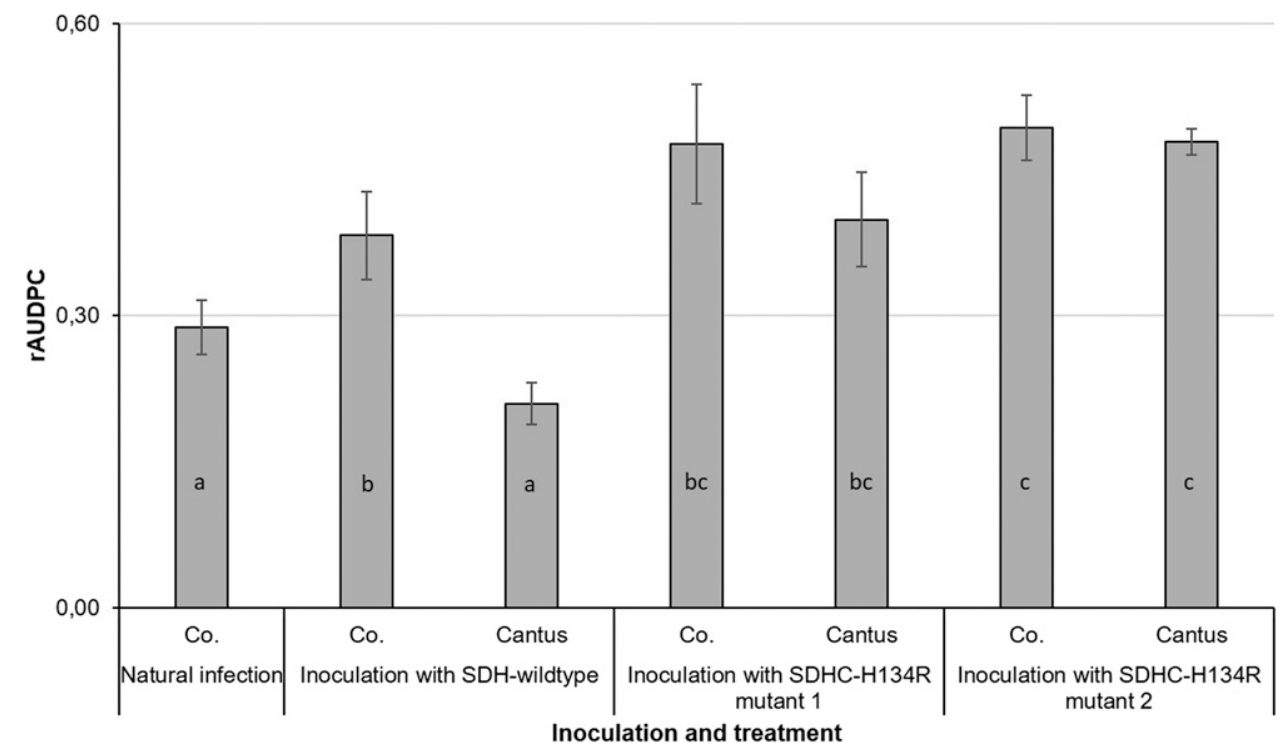

Fig. 5. Relative area under the disease progress curve (rAUDPC) values of three separate field inoculations with different Alternaria solani isolates-one succinate dehydrogenase (SDH) wild type and two SDHC-H134R mutants (isolate 628 is SDHC-H134R mutant 1 and isolate 687_1 is SDHC-H134R mutant 2) -and a natural infection in the field trial in 2017, using potato cultivar Lady Amarilla. For each inoculation, the mean rAUDPC values (calculated from four repetitions) of the untreated control (Co.) and the treatment with the SDHI fungicide Cantus ( $500 \mathrm{~g}$ of boscalid $/ \mathrm{kg}$ ) are shown. Bars marked with the same letter are not significantly different according to the post hoc Dunnett T3 test $(\alpha=0.05)$. Error bars show the standard deviation. 
observed. This was surprising in front of the presence of natural background infection levels that reached about half of the rAUDPC of successfully inoculated plots. One can possibly explain this by the outcompeting of natural wild-type $A$. solani by the mutant isolates, which started from a presumably much higher artificial inoculum. At the same time, Cantus proved potent in the control of early blight caused by wild-type $A$. solani even under increased pathogen pressure after inoculation. In general, the field trials in 2016 and 2017 confirm the results of the in vitro and greenhouse experiments with SDHC-H134R. It is therefore concluded that the frequent occurrence of the SDHC-H134R mutation in German potato fields could limit future use of SDHI fungicides against early blight in potato. Because the naturally occurring isolates additionally carried the F129L complex III mutation conferring partial QoI resistance, there seems to be the potential for multiple fungicide resistance in German A. solani populations. Landschoot et al. (2017a) tested the sensitivity of Belgian isolates against a mixture of boscalid (SDHI) and pyraclostrobin (QoI). The $\mathrm{EC}_{50}$ values of double mutants (SDHI and QoI mutation) were significantly higher compared with isolates with no mutation or a single mutation in the genome. Bauske et al. (2018b) also showed that there are differences between SDHI fungicides with different active ingredients like boscalid and fluopyram. Therefore, a detailed analysis of the sensitivity of the German A. solani population to SDHIs other than boscalid and also to alternative active ingredients is required. Additionally, the management and recommendation of plant protection measures should reconsider the high adaptive potential of A. solani populations to chemical plant protection.

\section{Acknowledgments}

We especially thank Andrea Klaus for technical assistance in the laboratory and the greenhouse, and we also thank the GHL Dürnast core facility for technical support during the greenhouse and field trials.

\section{Literature Cited}

Bartlett, D. W., Clough, J. M., Godwin, J. R., Hall, A. A., Hamer, M., and ParrDobrzanski, B. 2002. The strobilurin fungicides. Pest Manag. Sci. 58:649-662.

Bauske, M. J., Mallik, I., Yellareddygari, S. K. R., and Gudmestad, N. C. 2018a. Spatial and temporal distribution of mutations conferring QoI and SDHI resistance in Alternaria solani across the United States. Plant Dis. 102:349-358.

Bauske, M. J., Yellareddygari, S. K. R., and Gudmestad, N. C. 2018b. Potential impact of fluopyram on the frequency of the D123E mutation in Alternaria solani. Plant Dis. 102:656-665.

Campo, R. O., Zambolim, L., and Costa, L. C. 2007. Potato early blight epidemics and comparison of methods to determine its initial symptoms in a potato field. Rev. Fac. Nal. Agr. Medellín 60:3877-3890.

Christ, B. J., and Maczuga, S. A. 1989. The effect of fungicide schedules and inoculum levels on early blight severity and yield of potato. Plant Dis. 73:695-698.

Fungicide Resistance Action Committee. 2015. List of species resistant to SDHIs, April 2015. http://www.frac.info

Granovsky, A. A., and Peterson, A. G. 1954. Evaluation of potato injury caused by leafhoppers, flea beetles, and early blight. J. Econ. Entomol. 47:894-902

Gudmestad, N. C., Arabiat, S., Miller, J. S., and Pasche, J. S. 2013. Prevalence and impact of SDHI fungicide resistance in Alternaria solani. Plant Dis. 97:952-960.
Harrison, M. D., and Venette, J. R. 1970. Chemical control of potato early blight and its effect on potato yield. Am. J. Potato Res. 47:81-86.

Horsefield, R., Yankovskaya, V., Sexton, G., Whittingham, W., Shiomi, K., Omura, S., Byrne, B., Cecchini, G., and Iwata, S. 2006. Structural and computational analysis of the quinone-binding site of complex II (succinateubiquinone oxidoreductase): A mechanism of electron transfer and proton conduction during ubiquinone reduction. J. Biol. Chem. 281:7309-7316.

Kissling, E. 2003. BASF news release: EPA registers Endura and Pristine fungicides. BASF,Ludwigshafen, Germany.

Kuck, K. H., and Mehl, A. 2003. Trifloxystrobin: Resistance risk and resistance management. Pflanzenschutz-Nachrichten Bayer 56:313-325.

Kuhn, P. J. 1984. Mode of action of carboxamides. Pages 155-183 in: Mode of Action of Antifungal Agents. A. P. J. Trinci and J. F. Ryley, eds. Cambridge University Press, Cambridge, UK.

Landschoot, S., Carrette, J., Vandecasteele, M., de Baets, B., Höfte, M., Audenaert, K., and Haesaert, G. 2017a. Boscalid-resistance in Alternaria alternata and Alternaria solani populations: An emerging problem in Europe. Crop Prot. 92:49-59.

Landschoot, S., Vandecasteele, M., de Baets, B., Höfte, M., Audenaert, K., and Haesaert, G. 2017b. Identification of A. arborescens, A. grandis, and $A$. protenta as new members of the European Alternaria population on potato. Fungal Biol. 121:172-188.

Leiminger, J. H., Adolf, B., and Hausladen, H. 2014. Occurrence of the F129L mutation in Alternaria solani populations in Germany in response to QoI application, and its effect on sensitivity. Plant Pathol. 63:640-650.

Leiminger, J. H., and Hausladen, H. 2012. Early blight control in potato using disease-orientated threshold values. Plant Dis. 96:124-130.

Mallik, I., Arabiat, S., Pasche, J. S., Bolton, M. D., Patel, J. S., and Gudmestad, N. C. 2014. Molecular characterization and detection of mutations associated with resistance to succinate dehydrogenase-inhibiting fungicides in Alternaria solani. Phytopathology 104:40-49.

Odilbekov, F., Edin, E., Garkava-Gustavsson, L., Hovmalm, H. P., and Liljeroth, E. 2016. Genetic diversity and occurrence of the F129L substitutions among isolates of Alternaria solani in south-eastern Sweden. Hereditas 153:10.

Olaya, G., Zheng, D., and Köller, W. 1998. Differential responses of germinating Venturia inaequalis conidia to kresoxim-methyl. Pestic. Sci. 54:230-236.

Pasche, J. S., Wharam, C. M., and Gudmestad, N. C. 2004. Shift in sensitivity of Alternaria solani in response to QoI fungicides. Plant Dis. 88:181-187.

Rehfus, A., Strobel, D., Bryson, R., and Stammler, G. 2018. Mutations in sdh genes in field isolates of Zymoseptoria tritici and impact on the sensitivity to various succinate dehydrogenase inhibitors. Plant Pathol. 67:175-180.

Rotem, J. 1994. The Genus Alternaria: Biology, Epidemiology, and Pathogenicity. American Phytopathological Society, St. Paul, MN.

Shaner, G., and Finney, R. E. 1977. The effect of nitrogen fertilization on the expression of slow-mildewing resistance in Knox wheat. Phytopathology 67: 1051-1056.

Teramoto, A., Meyer, M. C., Suassuna, N. D., and da Cunha, M. G. 2017. In vitro sensitivity of Corynespora cassiicola isolated from soybean to fungicides and field chemical control of target spot. Summa Phytopathol. 43:281-289.

Van der Waals, J. E., Korsten, L., and Denner, F. D. N. 2003. Early blight in South Africa: Knowledge, attitudes and control practices of potato growers. Potato Res. 46:27-37.

Wharton, P., Fairchild, K., Belcher, A., and Wood, E. 2012. First report of in-vitro boscalid-resistant isolates of Alternaria solani causing early blight of potato in Idaho. Plant Dis. 96:454.

Yin, Y. N., Kim, Y. K., and Xiao, C. L. 2011. Molecular characterization of boscalid resistance in field isolates of Botrytis cinerea from apple. Phytopathology 101:986-995. 